\title{
3D Plants Reconstruction based on Point Cloud
}

\author{
Lanling Zeng ${ }^{\mathrm{a}}$, LingLing Zhang ${ }^{\mathrm{a}, *}$, Yang Yanga,, Wei Zhang ${ }^{\mathrm{b}}$, Yongzhao Zhan ${ }^{\mathrm{a}}$ \\ ${ }^{a}$ School of Computer Science and Comunication Engineering, Jiangsu University, Zhenjiang, 212001, China \\ ${ }^{b}$ Department of Information and Comunication, Jiangsu Wuzhong Secondary Vocational School, Suzhou, 215000, China
}

\begin{abstract}
Plant modeling based on point cloud is more difficult than others because of the noise-edge bonding. We present a method called the limited detail multi-density plants modeling to address these problems mentioned above. The core of the proposed method is a point-tosurface approach, which constantly refines the density of points with original RGB information to generate the fuzzy surface. Compared to conventional plants mesh models, the primary advantage of our approach is that it does not compute the steps of texture and illumination, and saves rendering time and storage space. We validate our method with both leaves and simple plants, and demonstrate that the limited detail multi-density point reconstruction is feasible, and it can generate good results with fast speed while using less storage space.
\end{abstract}

Keywords: plants modeling; the limited detail multi-density; point-to-surface

(Submitted on November 12, 2017; Revised on November 30, 2017; Accepted on December 20, 2017)

(C) 2018 Totem Publisher, Inc. All rights reserved.

\section{Introduction}

Plants play an important part in the natural landscape. Different types of plants have different structures and are structurally complex. During everyday life, plants are an indispensable part of virtual reality, computer games, film and television animation, as well as garden design systems. Thus, with the advent of image and 3D point cloud plants raw data acquisition technology, how to combine the early graphics-based plants modeling method with modern 3D point cloud modeling and human-computer interaction technology, efficient and convenient to complete plants modeling, will promote the development of plants modeling theory and practical application. However, accurate and efficient reconstruction of plants remains an open problem.

Various attempts have been made to present 3D plants modeling from different sources. Many algorithms play an important role in the reconstruction of plant models, such as fractal [10], L-system [12], image-based [18], sketch-based [9] and texture-lobes, as they have [6] been successfully applied to create plant modeling. In addition, the algorithm based on point cloud plant models have become more and more popular. However, there is some flaw in the adhesion between plant leaves and the rate of plant reconstruction.

This paper presents a novel modeling method based on the multi-density points like the particle system [15]. Although the particle system is the most efficient way to render the large-scale grasses, trees, sky, and simulate the dynamic water and smoke, it has obvious deficiencies in the model details [2]. Our work was inspired by the particle system, and the points collected through Kinect are used to generate 3D plants with rich details. Mesh reconstruction and realistic rendering parts are ignored in our work.

We introduce a method of the limited detail multi-density point reconstruction that solves the adhesion between plant leaves and improves the speed of the model reconstruction. Given the unorganized and orientated points of plants, we firstly delete the outliers and noise. However, the basic points' model will generate adhesion due to the speed and accuracy of scanning or other reasons. Then, we adopt an approach of L2-median with a color regularization to simplify the points and 
obtain the sparse point cloud, so the problem of adhesion between plant leaves is solved by iteration. Next, we use the multidensity method to add more points into point cloud based on neighborhood RGB information. Finally, we adjust the color of plant reconstruction and find that plant reconstruction models after color adjustment are closer to the nature shape.

The basic reference geometry model forms after the completion of the scan reconstruction, due to external factors, such as if the scanning speed is not uniform and if the scanner resolution is not high, or other factors. These problems will appear in the case of adhesion between the branches and leaves, especially at the lobes in the same leaf. In view of the fact of adhesion, an improved limited detail multi-density point reconstruction algorithm is proposed for plant reconstruction, which is based on RGB information and uses the implicit function framework to deal with the problem of surface reconstruction.

The limited detail multi-density point reconstruction is based on the sparse point cloud. Taking into account sensory experience of the users and the speed of reconstruction, we utilize the sparse point cloud to increase the density with the color information and get the points' reconstruction models. The rate of the point cloud reconstruction is higher than the speed of grid reconstruction, and the value is widely used.

Due to the dim light in the room and the poor resolution of the scanner, these factors could cause the overall color models to not be quite true to the original and appear gloomier than the real plants in the original environment. According to the above flaws, we perform a method of color adjustment on plant models after density. The results are more natural and close to the original plants after color adjustment.

In summary, our main contributions include:

- We develop the algorithm of L2-median with a regularization based on RGB information, which can separate the adhesive on the original point cloud as well as obtain the sparse points.

- We develop a limited detail multi-density point reconstruction algorithm, which is based on the limitation of human recognition and generates the fuzzy surface through refining the density of points to reach the purpose of surface.

\section{Related Work}

We summarize previous works in the developments of plants modeling.

\subsection{Graphic-based plants modeling}

Graphic-based techniques use procedural approaches, L-system, fractal, as well as reference axis techniques [16]. Okabe and Owada extend these approaches by presenting a system for designing 3D models of botanical trees quickly and easily using freehand sketches or additional example-based editing operations.

\subsection{Image-based plants modeling}

Image-based methods reconstruct plants with one or several image samples. Shlyakhter et al. present an approach for creating a visual hull of the tree and constructing the tree skeleton, where they utilize 4-15 registered images and find an approximation to the medial axis of the tree's visual hull. They introduce L-system to fit the smaller branches and leaves. Reche et al. [14] use a set of calibrated images to determine the volumetric shape of a given tree. The volume is divided into grid cells, which is assigned a set of view-dependent texturing algorithms.

\subsection{Point-based plants modeling}

Quan et al. [13] use a hand-held camera to capture images of plants at different views, and then apply a standard structure from motion technique [5] to recover the camera parameters and 3D point cloud. They segment 3D points and 2D images into individual leaves and extract a generic leaf model that can be used to fit all the other leaves. This approach is developed by Tan et al. [11], which can be used to construct more complex trees. The results are realism but the process is complex.

Bucksch [1] presents a reconstruction pipeline for recovering branch structure of trees from laser scanned points data. Yotam Livny et al. [20] perform an active laser scanning of natural vegetation and achieve the original point cloud where the trees could be created. They use a series of global optimizations to make the skeletal structures fit in the noisy points data. The original point cloud could be obtained from images of trees at different views [17]. 


\subsection{Three-dimensional data from depth camera}

It is involved in plant modeling. The born of Kinect is made for people to get the 3D data easier than before. Shen [7] distinguishes some special 3D objects around the model library by the characteristics of the scanned objects. Yan [21] explores the internal structure by uncovering the hidden blade when using the depth camera.

\section{Overview}

We carry out the limited detail multi-density point reconstruction, which involves de-noising, sparsity, reconstruction and color adjustment.

In the first step, we use Kinect to scan plants of overall basic geometry. The obtained results are shown in Figure 1(a) and 1(b). The original points' model of plants has adhesion at the position of bifurcation, as well as noise spots around the leaves. Furthermore, the complexity of plants itself is different, and so the adhesive degree of the reconstructed plants models will be different.

Next, we use the de-noising algorithm based on K-means clustering to remove the noisy points, and the result is shown in Figure 1(c). Then, we perform local L2-median algorithm to the sparse point cloud, which can get a small data set as shown in Figure 1(e). Compared to the Poisson reconstruction algorithm [3,4] , which shows the adhesion and wrong connection at the bifurcations, our method of the limited detail multi-density point reconstruction can solve the problem of adhesion and wrong connection at the bifurcations, as shown in Figure 1(d) and (g). In this algorithm, we perform the increasing density operation by judging the European distance between any two points. In addition, we use the distance parameter $\gamma$, which can be used as a threshold to decide the density of points. The entire plants point modeling reconstruction can be generated through the continuous iteration. Finally, the phenomenon of adhesion between plant leaves has been solved, but the overall color of the reconstructed plants is different from the original plant color. We introduce a color-adjusted approach to get the final result shown in Figure 1(h), which is more authentic and closer to the original plants in Figure 1(a) compared to Figure 1(f).

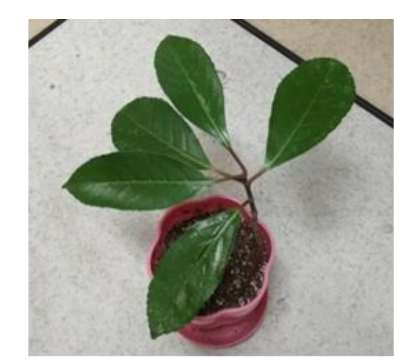

(a)Photo

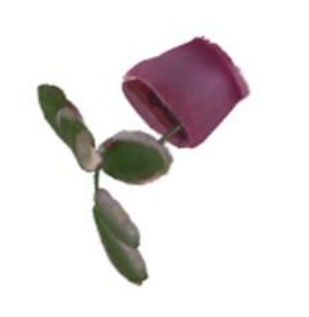

(d) The original model of 'sticky' (e) the points cloud model after sparse

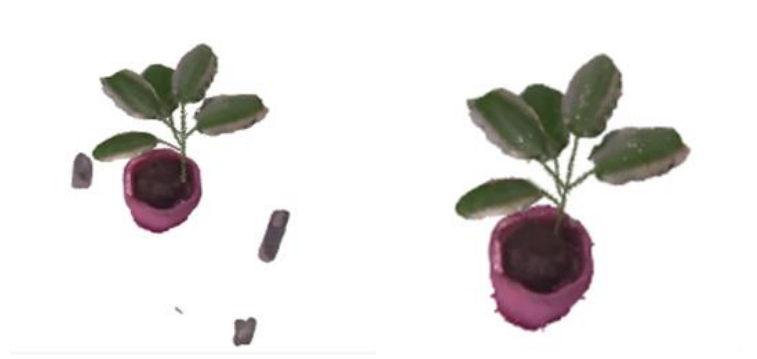

(b) Original model with noise

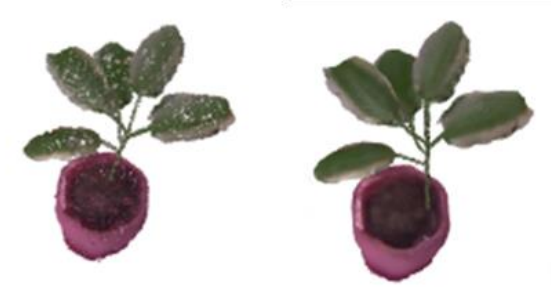

(f) the points cloud model after density (c) Original model without noise

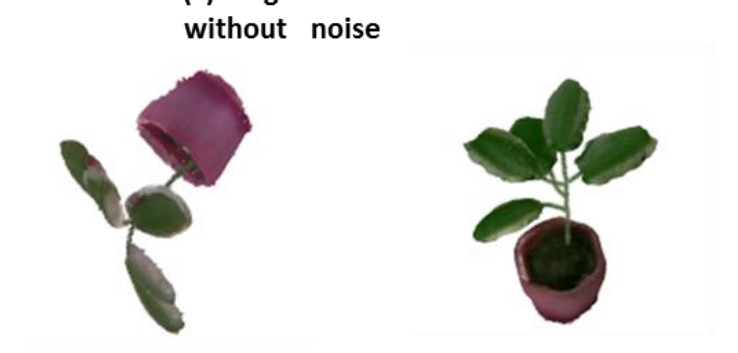

(g)the model of 'Not sticky' (h)the model of 'Not sticky' after sparse and density after color adjustment

Figure 1. The complete process of leaves reconstruction

\section{Point Cloud Extraction and Simplification}

\subsection{Point cloud extraction and matching}

The initial plants are scanned by Kinect with depth and RGB information as shown in Figure 2(a), which is the original plant photo. We firstly fix the Kinect, and then plants are placed on a rotating chair and scanning site as much as possible to avoid complex background. The purpose of the setting is to reduce the noisy points effectively. After we record the 3D data of 
each point, the background information by preconditioning is separated, and then we can get the whole scanned model, as shown in Figure 2(b).

In order to obtain the 3D data integrally, we need to first convert the depth data from the pixel map. It is well known that pixel data as $u=(x, y)$ are collected in the pixel map $\boldsymbol{D}_{i}(u)$. Then, the 3D coordinate $\boldsymbol{v}_{i}(\boldsymbol{u})$ can be received by the calculation of the built-in matrix $\boldsymbol{K}$. The formula is shown in Eql(1). Finally, the normal vector $\boldsymbol{n}_{i}(u)$ of this point can be shown in Eql(2) by using formula (2).

$$
\begin{gathered}
\boldsymbol{v}_{i}(u)=\boldsymbol{D}_{i}(u) \boldsymbol{K}^{-1}[u, 1] \\
\boldsymbol{n}_{i}(u)=\left(\boldsymbol{v}_{i}(x+1, y)-\boldsymbol{v}_{i}(x, y)\right) \times\left(\boldsymbol{v}_{i}(x, y+1)-\boldsymbol{v}_{i}(x, y)\right)
\end{gathered}
$$

During scanning, we use the conventional algorithm of the ICP (Iterative Closest Point) algorithm to track the camera. We can obtain the original data by moving the camera. It is one of precise registration algorithms for a set of points. Generally, for each scanning point, a corresponding point is received as the closest Euclidean distance in the model, and then the method is continuously iterated to minimize the objective function.

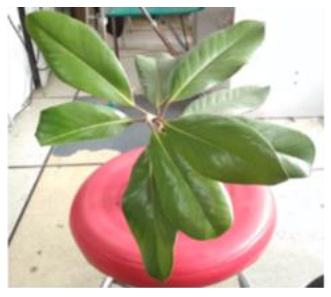

(a)the original photo

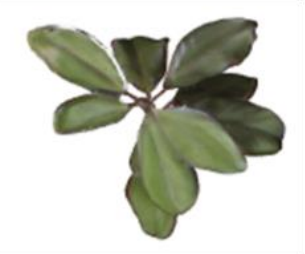

(b)the original model after the reconstruction without processing

Figure 2. The process of the leaves reconstruction

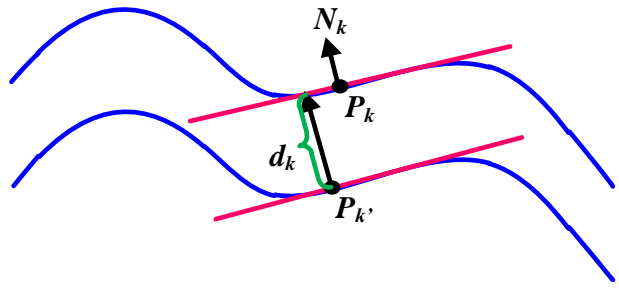

Figure 3. ICP Algorithm

As shown in Figure 3, we suppose the original point named $\boldsymbol{P}_{K}$ in the area $\boldsymbol{R}$, and $\boldsymbol{P}_{K^{\prime}}$ is the destination point that must correspond to $\boldsymbol{P}_{K}$ in the same region of $\boldsymbol{R}$. The core of ICP algorithm is to find an excellent transformation matrix $\boldsymbol{T}$ that allows the minimum distance $d$ between two points. In the recently proposed technology of Kinect Fusion, the absolute posture $\boldsymbol{T}_{\mathrm{g}, k}$ of the depth camera in the world, which means the current frame $k$ relative to the global frame $g$, is given by calculating the minimum total error for all corresponding points, as shown in Eql (3).

$$
E\left(T_{g}, k\right)=\sum_{\Omega \neq \Phi}\left\|\left(T_{g}, k V_{K}(u)-\hat{V}_{k-1}^{g}(u)\right)^{\mathrm{T}} \hat{N}_{k-1}^{g}(\hat{u})\right\|_{2}
$$

Where $\boldsymbol{T}_{g, k}$ is a pose matrix for $4 \times 4 \cdot V_{K}(u)$ is the $3 \mathrm{D}$ coordinate of current frame $\boldsymbol{P}_{K} \cdot \hat{\boldsymbol{V}}_{K-1}^{g}(\hat{u})$ is the $3 \mathrm{D}$ coordinate of model frame $\boldsymbol{P}_{K^{\prime}}$, and $\hat{\boldsymbol{N}}_{K-1}^{\mathrm{g}}(\hat{u})$ is the normal vector of the corresponding models points. Finally, the optimized problem is transformed into the least square optimization, and the optimal solution is obtained by calculating a linear equation.

Point cloud from the ICP algorithm is the closest data to the original point cloud value. As shown in Figure 4, the point models of plants are displayed in the experiment. The excess circle part in the Figure is the experimental bench, which can be removed in the post-processing.

\subsection{De-noising on the original point cloud}

Many outliers and noisy points exist in the original models, especially the adhesion between leaves and branches, which can lead to the wrong result. To address this problem, we firstly use noise-free operation, which can remove the outliers and the noisy points in the original models.

In 2016, Su and Ma [19] proposed a noise-free method based on K-means clustering. In the scattered cloud processing, the K-means algorithm can quickly classify the point cloud data to obtain a compact and independent cluster. We compare 
the distance from each point in the cluster to the center of the cluster, and then the estimated curvature relation of the analysis point will help us find the noisy points and then remove them.

The original input data set is $\mathrm{P}\left(x_{1}, x_{2}, \ldots x_{N}\right)$, and each $x_{i}$ is a 3-dimensional vector. The purpose of $\mathrm{K}$-means clustering is to divide the original data into class $\mathrm{K}, \mathrm{S}=\left\{s_{1}, s_{2}, \ldots s_{K}\right\}$ under the condition of the given group number $\mathrm{K}(\mathrm{K}<=\mathrm{N})$. Given an unorganized and orientated set $\mathrm{P}$, we carry out the following definition for noise-free that leads to an optimal set of the denoised points. The expression for the minimum is shown in $\operatorname{Eql}(4)$.

$$
\operatorname{argmin} \sum_{i=1}^{K} \sum_{x_{j} \in S_{i}}\left\|\mathrm{x}_{j}-\mu_{i}\right\|^{2}
$$

Where $\mu_{i}$ represents the average of the class $S_{i}$.

We can obtain the $\mathrm{S}=\left\{p_{i}, \mathrm{i}=1,2, \cdots n\right\}$ with the above noise-free method, which is listed as following:

- Step1. Calculate the Euclidean distance of each point $p_{i}$ in the cluster to cluster center $\mu$.

- Step2. Calculate the estimated curvature of each point by the curvature estimation algorithm and obtain the average of all points curvature as $\operatorname{Eql}(5)$.

$$
\bar{k}=\frac{1}{n} \sum_{i=1}^{n} k_{i}
$$

- Step3. Give the value of $\omega(\omega>1)$, which is used to define the degree of noise removal. When the value of $\omega$ is smaller, the greater degree of noise is reduced. (It is possible to remove the non-noise point cloud.)

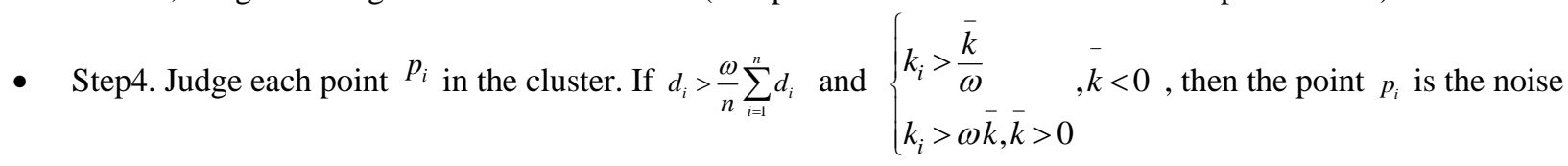
point. Then, remove the point.

The de-noised point cloud data can be obtained when the de-noising algorithm is adopted. It is shown in Figure 5.

\subsection{Simplification on the de-noised point cloud}

After de-noising, there still exists the problems of adhesion between the stems and the blades, and another problem is color distortion of the edge points, which come from the error sensitive Kinect [8].

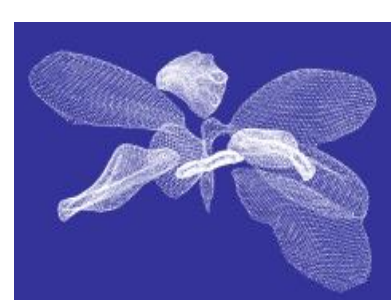

Figure 4. The points of leaf

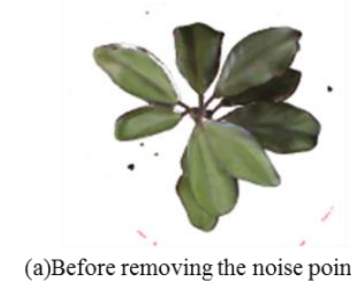

Figure 5. Point cloud of removing noise point operation

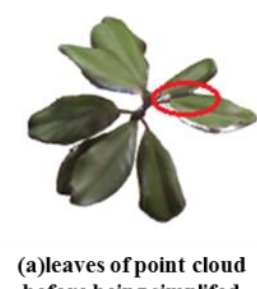

before being simplifed

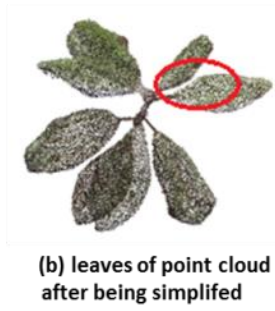

(b) leaves of point cloud
after being simplifed

Figure 6. The disappearance of bonded portions

Therefore, we have to adopt some measures to handle these adhesion and cross-color points. We investigate the following definition for L2-median simplification. The first term is a localized L2-median of inputting points set P, which has no outliers and noise, and the second term is the color statistical function $\phi(r, g, b)$. Due to the oneness of the blade, a lot of different color points in the blade can be obviously found. We count the color data to find that most points' color data fall on a certain interval. Then, we introduce a parameter $\beta$ to control the range of the color data. The advanced formal is shown in $\operatorname{Eql}(6)$.

$$
E(\chi)=\sum_{p \in P}\|\vec{V}(p)-\nabla \chi(p)\|^{2}+\beta \cdot \phi(r, g, b)
$$


Through the change of parameter $\beta$ in constraint items, point cloud is gradually diluted.

We have performed a validation of this approach by iteration. Over some lobes leaves and simple indoor plants tested, the average iteration times are between 3 to 6 and depend on the complexity of the point cloud. Figure 6 illustrates that there are different sparse points with different iteration times.

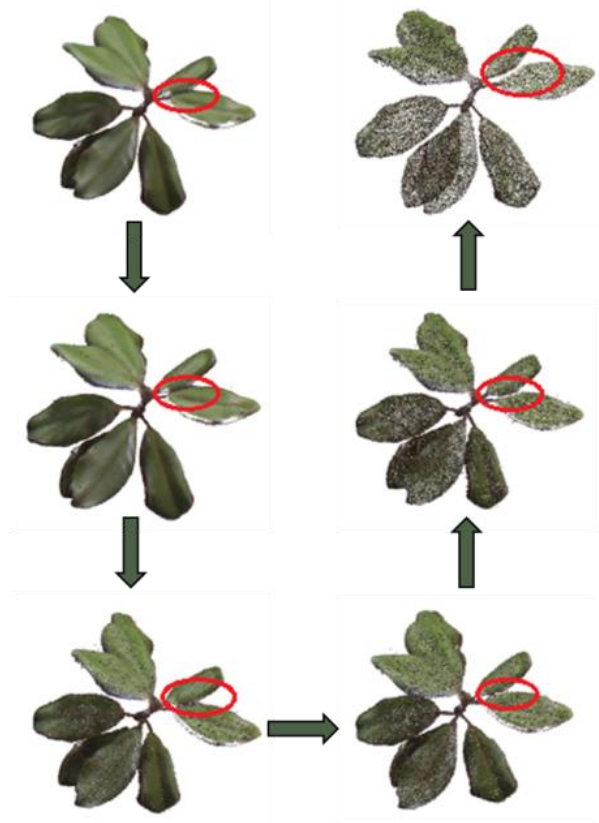

Figure 7. Gradient deleting the points
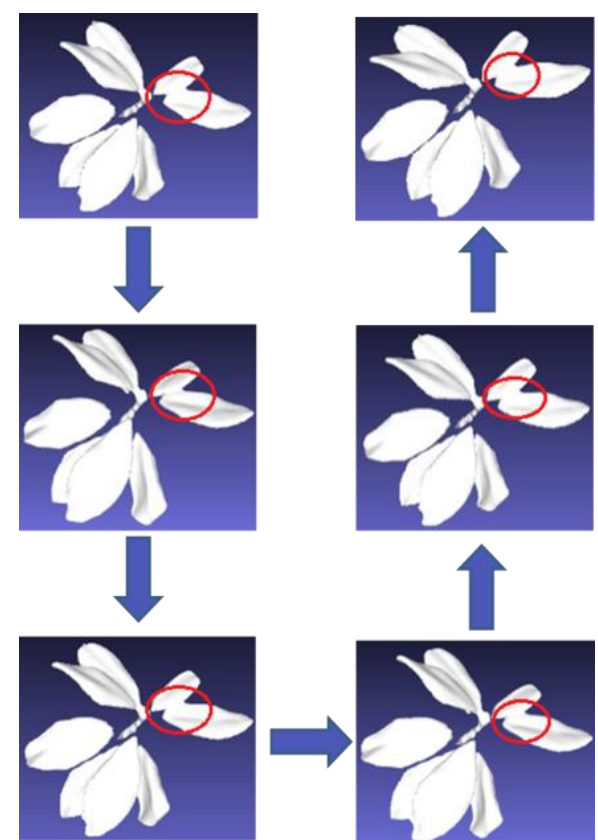

Figure 8. Reconstruction of points

In the first place, we use formula (6) to get the point models after each step of the sparse. However, the key threshold cannot be determined accurately and can only estimate the approximate range. So, we decrease the point color value on uniform gradient and the experimental results for every sparse are shown in Figure 7.

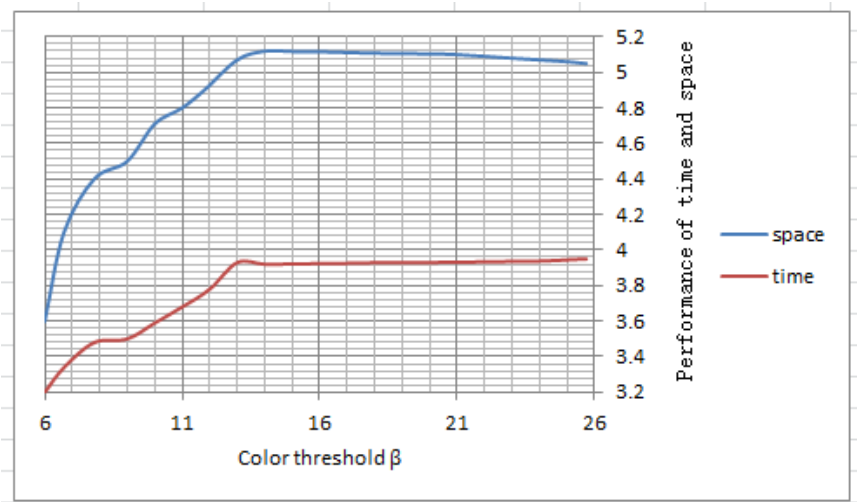

Figure 9. The trend using of time and space

After that, corresponding to Figure 7, the mesh models by Poisson surface reconstruction algorithm are shown in Figure 8. It is the reconstruction of the critical value $\beta_{\xi}$ in fifth leaf according to the arrow direction, and we can find that deleting some point cloud will not affect the results of the reconstruction when the critical value is more than $\beta_{\xi}$.

Observing the mesh models, especially the fifth blade in the direction of the black arrow, we find that it is very similar to the shape of the original one. That is to say, when the sparse parameter is around a range, deleting some points will not affect the result of the reconstruction, and the adhesions between leaves still exist. 
When processing the temporal and spatial parts of reconstruction, we can get the performance trend of our approach. Figure 9 illustrates that the time and space performance are optimized to a certain extent by using the sparse point cloud in the premise without affecting the reconstruction result.

\section{The limited Detail Multi-Density Point Reconstruction}

Compared to the mesh models, we find that the split parts are also adhesive together between two adjacent leaves after the mesh reconstruction, which has been separated by using formula (6) in the sparse process. The adhesive parts in mesh reconstruction are shown in Figure 10 marked in red ovals. Furthermore, the mesh reconstruction algorithm will spend a lot of time and space performance if we build the actual large natural landscape.

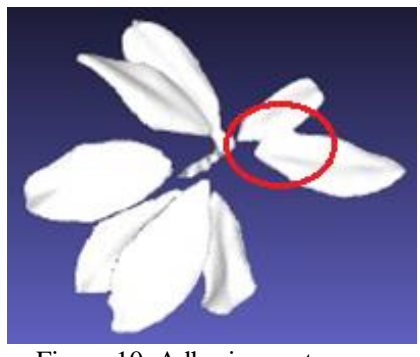

Figure 10. Adhesive parts

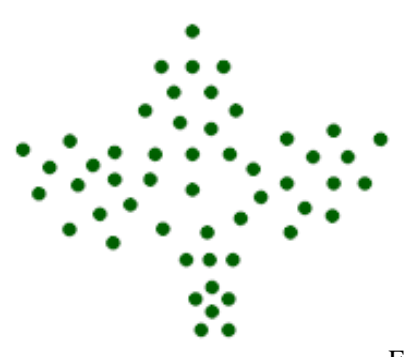

Figure 11. Multiple density point cloud

Due to the above problems of mesh reconstruction, we propose a limited detail multi-density point reconstruction algorithm. This algorithm is a 'special reconstruction' that is based on the fuzzy recognition of human eyes and sacrifices some normal points of the model. The principle of this special algorithm is based on the fact that the eyes will not clearly see the distance between points until the density of point cloud reaches a certain level. The aim of reconstruction is that the density of points can be made to synthesize a smooth surface by using the visual error.

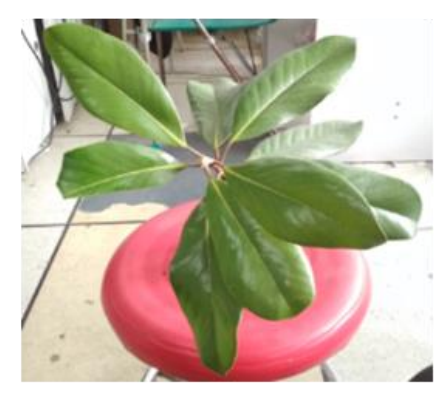

(a) the leaves photos

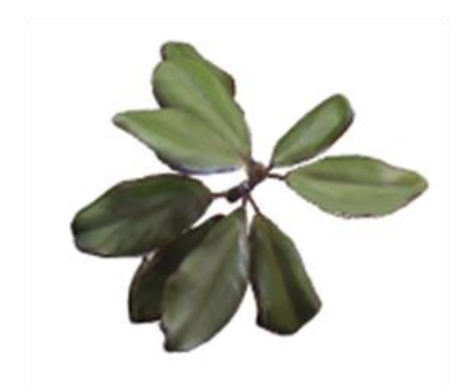

(b) the model of points cloud after the density

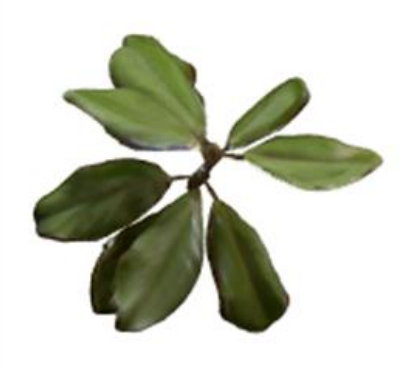

(c) color adjustment after the density

Figure 12. Color adjustment based on point cloud after the encryption

Figure 11 simply shows the process of the limited detail multi-density point reconstruction. Figure 11(a) is the original plant photo. The middle image in Figure 11(b) represents the point cloud after the first limited detail multiply-density, and the right image in Figure 11(c) is the point cloud after the second limited detail multiply-density with colour correction. Specifically, we consider the problem of adding more points into the location range, where there is the minimum total Euclidean distance to a set of points. As a result, this optimization problem is similar to linear sparse interpolation. Firstly, we define a local range with a finite support radius $r$ and then compute Euclidean distance of each two adjacent points. If the sum of all distances is greater than the given threshold, we will add a new point $x_{i}$ into the set and the value of the new point in this local range based on $\operatorname{Eql}(7)$.

$$
x=\operatorname{argmin} \sum_{i=1}^{k} \sum_{j=1}^{n}\left\|x_{i}-\mathrm{p}_{j}\right\|+R_{\text {smooth }}
$$

Where $k$ represents the number of $x_{i}$, and $j$ represents the point in the neighbourhood. It will generate more density points through applying Eql(7) along the first item, where the new points likely join the place of greater density. In order to 
avoid such clustering problems, we add another item $R_{\text {smooth }}$ in Eql(6), which keeps the new point from local center position and acquires an even distance between points. We set the value of semi-supervised and then new points are constantly added into the point cloud. Considering different scales, we use different densities of points to get the limited detail multi-density models where the density can be changed according to the distance.

The advantage of adopting the limited detail multi-density point reconstruction is that we can regulate the density of points in the models according to the dynamic distance between the perspective of the user and observation models. Compared to the current methods of mesh reconstruction, the multi-density of our method has more superiority, not only including small amounts of calculation, but also small storage capacities of models. Therefore, the method of the limited detail multi-density point reconstruction is a new and effective method for plant modeling.

\section{Adjust the color of point cloud}

Although we adopt a series of methods to deal with problems, such as noise and adhesion, and some of these problems have been solved, there are still some flaws in the whole reconstruction results, like the overall color of plant reconstruction. In order to solve the problem, we use a method of colour adjustment. Figure 12(a) is the original plant photo, Figure 12(b) and Figure 12(c) are two 3D results, and the colour of plant reconstruction in Figure 12(c), which is handled by colour adjustment, is significantly closer to the colour of plants in the original environment as shown in Figure 12(a). In order to attain the effect, firstly, we sample the colour information in the original two-dimensional image and only divide the colours of plants into two major categories: the main colours just from the leaves and stems. We then compute the mean values of leaves and stems alternatively. Secondly, we cluster two classes of 3D points based on colour information similar to the first step, and then change the points colour information using the $2 \mathrm{D}$ mean values with a predefined threshold, which can make the results more realistic. We modify the values of $\mathrm{R}, \mathrm{G}$ and $\mathrm{B}$ respectively with the new threshold, and when this condition is satisfied, the color of the point cloud is closer to the mean value of the colour of the original plants leaf. We then stop adjusting and finally get the result shown in Figure 12(c).

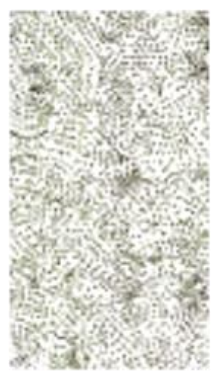

(a)Doubling

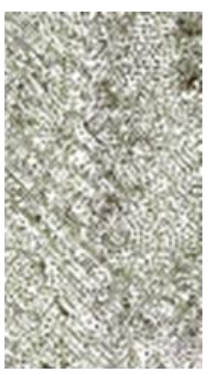

(c)Quadrupling (d)Quintupling

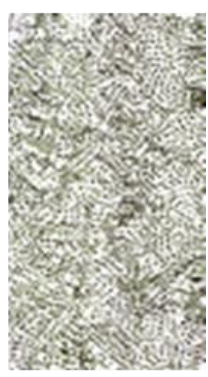

(b)tripling

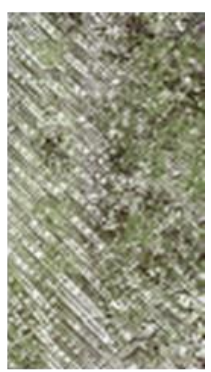

Figure 13. Density comparison

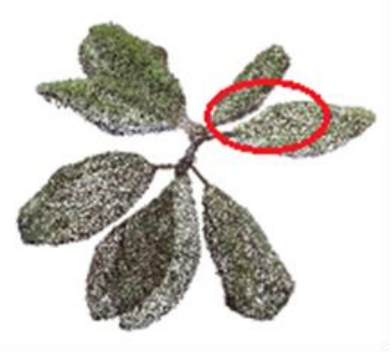

(a)the model of point cloud after sparse

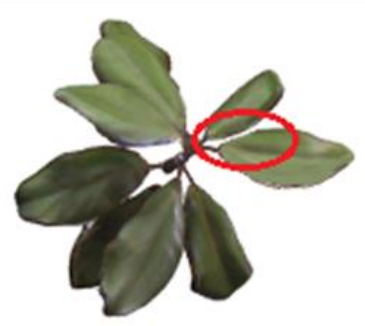

(b) the model of points cloud after the density

Figure 14. The result

\section{Results}

The density of points in our method generally tend to generate models with limited points. Figure 13 shows the results of the models under different densities. The color of new points added among a local space is set by an intermediate color of neighbors. Comparing the initial physical models with the multi-density models at the same ratio, human eyes can identify the original point cloud, and can't see the gap between the points. Furthermore, we can see the whole as a continuous surface 
with the density increased. Figure 14 shows some experimental results and the split parts are not bonded again based on our algorithm.

The limited detail multi-density point reconstruction algorithm is based on the trend of the human eyes' limitation in theory. The standard quality for results depends on the observer's sensory experience. Because depth data and RGB information are only needed to build whole blade models without calculating the normal vector, the file size of models and the speed of reconstruction have qualitative improvement. There will be an extremely high value for industrial applications.

Other blade models are shown in Figure 15, 16 and 17.

Figure 15 shows that the point cloud of these blades gradually decreased and finally, the problem of the adhesion between blades and leaves is resolved. Apart from this, the points with different color on the boundary have also been reduced. Figure 15(b) is the initial point cloud with adhesion between adjacent leaves, as well as between leaves and petioles. We use the sparse method to decrease the point cloud of the plants uniformly, and the sparse results can be seen intuitively in Figure 15 (c-e) with different degrees.

Figure 16 shows the results by using conventional mesh reconstruction, particularly the Poisson surface reconstruction algorithm. We set the point color parameter as a weight for the reconstruction to control these blade models' rebuilding with different number of points. During each step of reconstructing, we can find that the model with the sparse points is similar to the original model when the point color value is controlled in a special range of the key threshold $\beta_{\xi}$. Users typically cannot see the subtle difference in them, as shown in Figure 16(b) and 16(c). However, when the parameter is less than the key threshold $\beta_{\xi}$, more points have been decreased and the corresponding mesh reconstruction models have many holes. As a result, the reconstruction process has failed. The performance of time and space are displayed in Figure 16(d). In the premise of not affecting the models' effect, comparing 15 to 16 , an error will easily occur if we perform a mesh rebuild. Therefore, it makes our research more meaningful. The reconstruction of our method with sparse has a better performance.
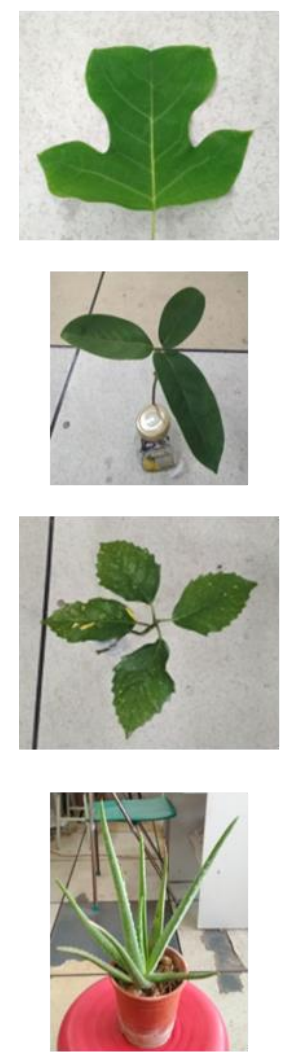

(a) The blade
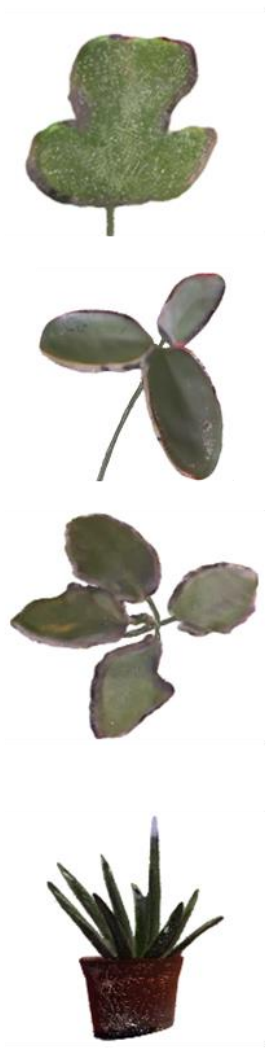

(b)Original points
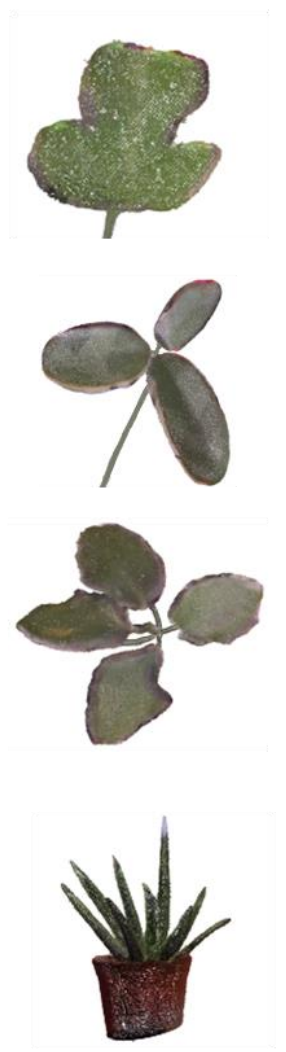

(c) Decrease for once
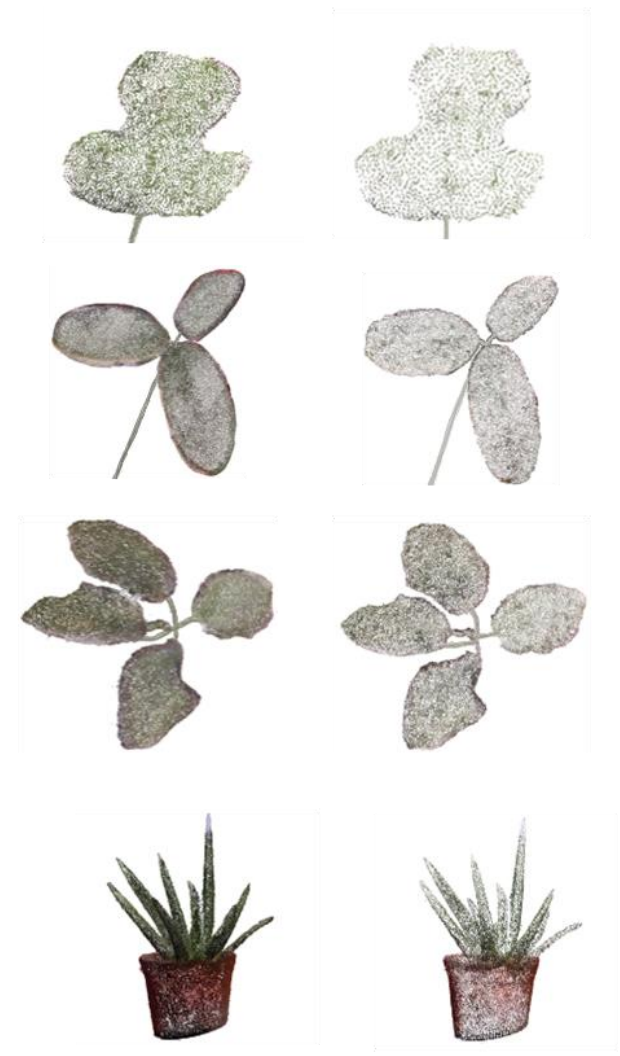

(d)Decrease for twice (e)Decrease for thrice

Figure 15. The spare of other blade models 

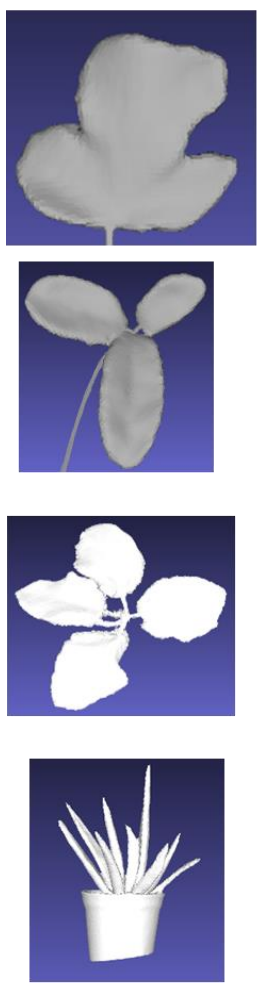

(a) Original models
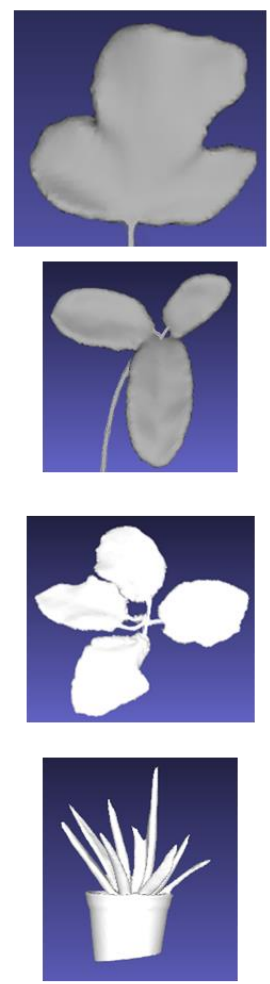

(b) Models with $<\beta$,
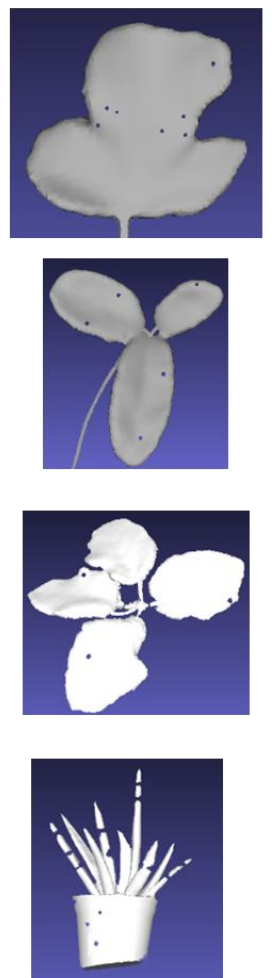

(c)Models with $<\beta$
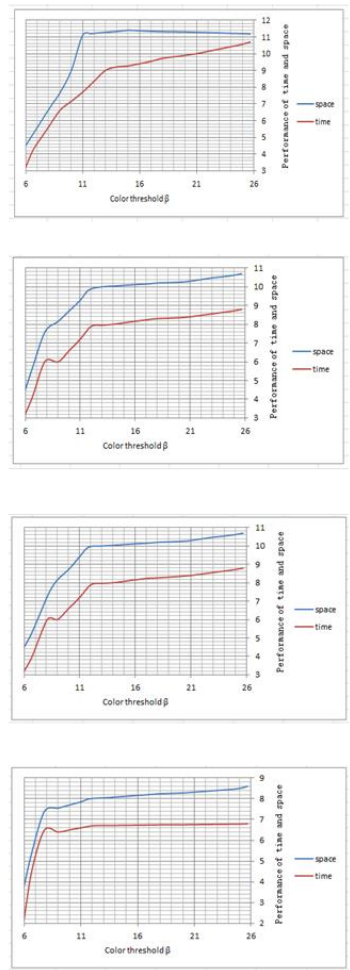

(d) The performance charts

Figure 16. The Poisson surface reconstruction with the sparse point cloud
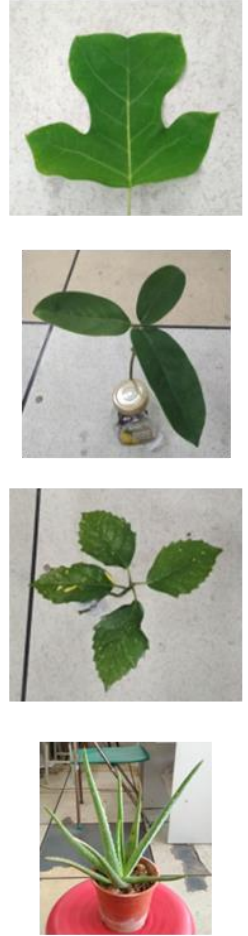

(a)The blade
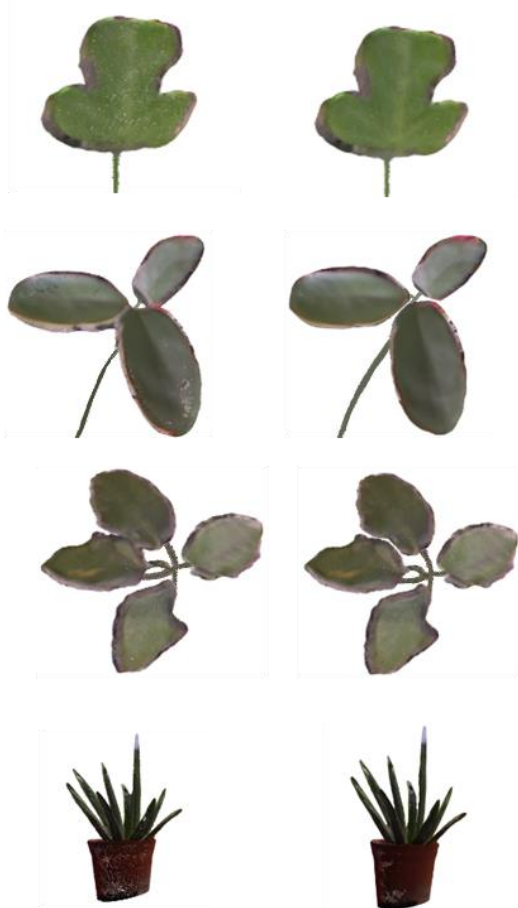

(b)Original models
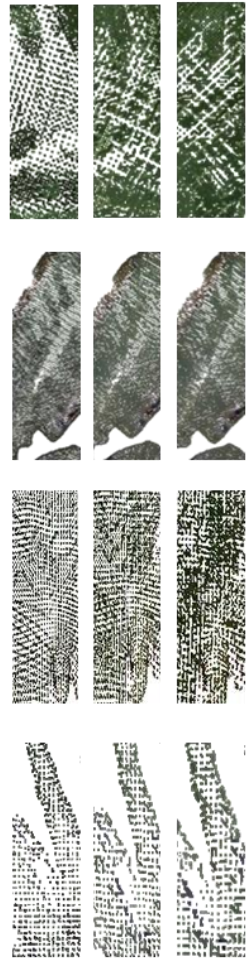

(d) the encryption before Plus smoothing item
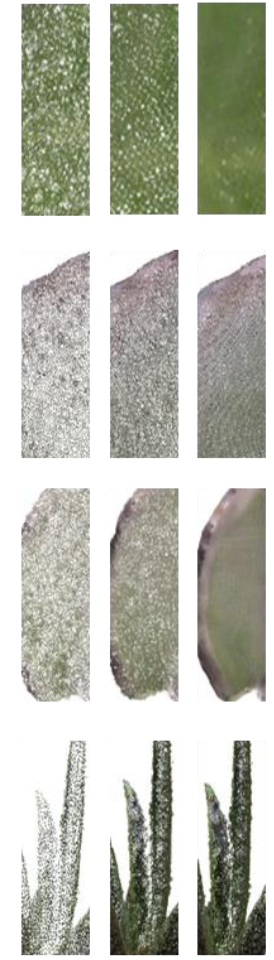

(e) the encryption after Plus smoothing item
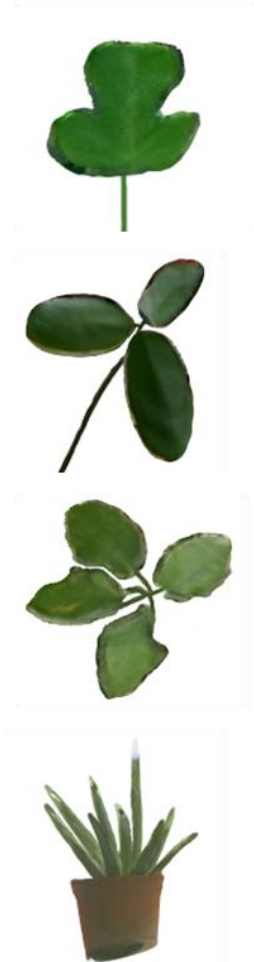

(f) color adjustment after the density

Figure 17. Comparison of experiment result 
In Figure 17(c), we get the blade models by using our method. When the models are scaled in the appropriate proportion, these points are dimmed as a surface. If point cloud is continuously increased, the aim of the fuzzy surface can be reached and the purpose of reconstruction can be realized. In addition, we don't find adhesion between adjacent leaves as well as between leaves and petioles. We solve the adhesion successfully. Figure 17(d) [22] shows the contrast in the same part of different densities in two times, four times and six times from left to right. Comparing Figure 17(d) and Figure 17(e), we can see that the plant point cloud in Figure 17(e) is even more uniform than that in Figure 17(d). The main difference is that the addition of a smoothing item $R_{\text {smooth }}$. Although we solve the adhesion between the leaves and the uniformity of the point cloud between the leaves by using our improved method, the color of plant models is considerably different from the original plant's in overall color, and our method is gloomy because of the precision of equipment and the ambient light. Therefore, we carry out the color-adjustment of plans models after multi-density. Finally, we get plant models that are closer to the original plants in Figure 17(f).

More detail data is shown in Table 1.

If the limited detail of the multi-density point reconstruction algorithm is applied to the actual scene, the size of plants become larger by mouse scrolling, and more points have joined the same speed in accordance. After that, the whole scene has been rendered as the favorable experience that is given to users.

Table 1. The data of Experiment

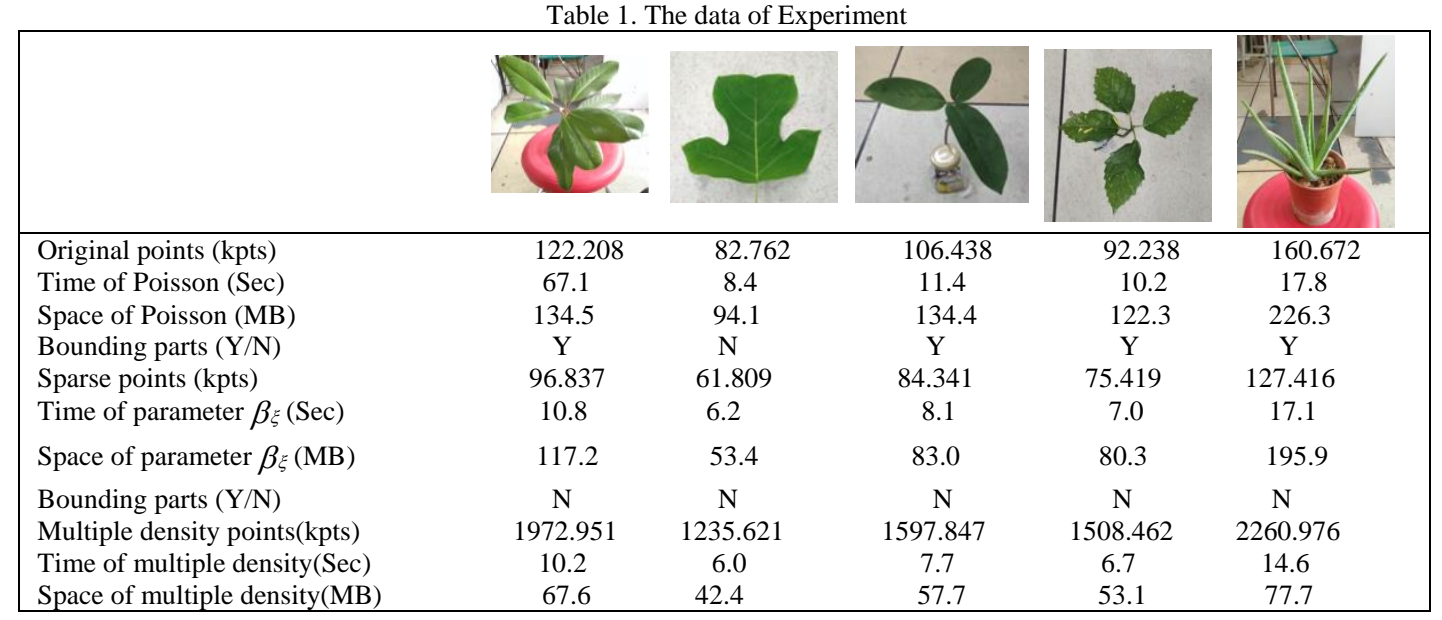

\section{Conclusions}

This paper improves the algorithm of plant reconstruction based on RGB information. Firstly, we use Kinect to scan and reconstruct plants modeling to obtain the original point cloud modeling. Then we adopt the de-noising algorithm based on $\mathrm{K}$-means clustering to remove the noisy points, and finally we obtain the ideal sparse point cloud modeling by using the L2median method.

On the basis of the sparse point cloud, a novel finite detail multi-density point reconstruction algorithm is developed. The idea of this algorithm is to start from the actual experience of the human eye, and constantly density its point cloud until the human eye cannot be identified. In the end, it forms a special point cloud based on limited detail 'fuzzy reconstruction'. Apart from this, we use a color-adjustment method for plants reconstruction after the encryption, and finally plant models that are closer to the original models are obtained. In addition to this, the plant models after reconstruction are more realistic.

\subsection{Limitations}

A limitation of our approach is the color smoothness of the blade's edge after the reconstruction. The color of the blade edge is not smooth and the point cloud between the leaves is not uniform because the scanner does not have high accuracy or uniform scanning speed. We expect to use a high-quality scanner for uniform sampling, which will help solve this problem.

Another limitation is that the multi-density point reconstruction is carried out on the basis of the ideal sparse point cloud. The requirements of the ideal point cloud are high, not only to solve the adhesion problem, but also to ensure that it does not affect the reconstruction, so the regulation of parameter $\beta$ is particularly important. 


\subsection{Future work}

Although our work has in this paper was based on limited detail points, the infinite detail based on points is another direction that we might explore. For example, if we are collecting objects that do not have RGB information, like transparent objects, finally we successfully carry out modeling reconstruction and point density. If this is available, the scope of the technology that we proposed will be more extensive and will have a qualitative improvement.

\section{Acknowledgements}

The work described in this paper was supported by National Natural Science Foundation of China (Grant No. 61402205), National Natural Science Foundation of China (Grant No. 61601202), China Postdoctoral Science Foundation (Grant No. 2015M571688), Jiangsu University (Grant No. 13JDG085), Research Foundation for Talented Scholars of Jiangsu University (Grant No .14JDG038), and Natural Science Foundation of Jiangsu Province(Grant No. BK20140571).

\section{References}

1. A. Bucksch and R. Lindenbergh, "Campino - a Skeletonization Method for Point Cloud Processing," ISPRS Journal of Photogrammetry and Remote Sensing, pages 115-127, 2008

2. O. Deussen, C. Colditz, M. Stam minger and G. Drettakis, "Interactive Visualization of Complex Plants Ecosystem," IEEE Visualization, pages 49-53, 2002

3. M. Kazhdan, M. Bolitho and H. Hoppe, "Poisson Surface Reconstruction," In Proceeding of The fourth Eurographics symposium on Geometry processing, pages 61-70, 2006

4. M. Kazhdan and H. Hoppe, "Screened Poisson Surface Reconstruction," ACM Transactions on Graphics, pages 61-70, 2013

5. M. Lhuiller and L. Quan, "A Quasi-dense Approach to Surface Reconstruction from Uncalibrated Images," IEEE Transactions on Pattern Analysis and Machine Intelligence, pp:418-433, 2005

6. Y. Livny, S. Pirk, Z. L. Cheng, F.L. Yan, O. Deussen, D. Cohen-Or and B.Q. Chen, “Texture-Lobes for Tree Modelling,” ACM Transaction on Graphics, pages 76-79, 2011

7. Y. Livny, F. Yan, M. Olson, B. Q. Chen, H. Zhang and J. El-Sana, "Automatic Reconstruction of Tree Skeletal Structures from Point Clouds," ACM Transactions on Graphics, pages 81-95, 2010

8. R. A. Newcombe, S. Izadi, O. Hilliges, D. Molyneaux, D. Kim, A. J. Davison, P. Kohli, J. Shotton and A. Fitzgibbon, "KinectFusion: Real-time Dense Surface Mapping and Tracking," In Proceeding of IEEE international symposium, pages 127136, 2011

9. M. Okabe, S. Owada and T. Igarashi, "Interactive Design of Botanical Trees Using Freehand Sketches and Example-based Editing," In Proceeding of EUROGRAPHICS, 24:3, 2005

10. P. E. Oppenheimer, "Real-Time Design and Animation of Fractal Plants and Trees," Computer Graphics, pages 55-64, 1986

11. T. Ping, Z. Gang and W. E. Jingdong, "Image-based Tree Modeling," In Proceeding of ACM SIGGRAPH 2007, Article 87, 2007

12. P. Prusinkiewicz and A. Lindenmayer, "The Algorithmic Beauty of Plants," Springer-Verlag New York, 1990

13. L. Quan, P. Tan, G. Zeng, L. Yuan, J. Wang and S. B. Kang "Image-based Plants Modeling," $A C M$ Trans.Graph.(Proc.SIGGRAPH), pp:772-778, 2006

14. A. Reche, I. Martin and G. Drettakis, "Volumetric Reconstruction and Interactive Rendering of Tree from Photographs," ACM Transactions on Graphics, pages 720-727, 2004

15. W.T. Reeves and R. Blau, "Approximate and Probabilistic Algorithms for Shading and Rendering Structured Particle Systems," ACM Transaction on Graphics, pages 313-322, 1985

16. P. D. Reffye, C. Edelin, J. Francon, M. Jaeger and C. Puech, "Plants Models Faithful to Botanical Structure and Development," Computer Graphics, pages 151-158, 1988

17. C. H. Shen, S. S. Huang, H. Fu and S. M. Hu, "Adaptive Partitioning of Urban Facades," ACM Transactions on Graphics, 6164,2012

18. I. Shlyakhter, M. Rozenoer, J. Dorsey and S. Teller, "Reconstructing 3D Tree Models from Instrumented Photographs," IEEE Computer Graphics and Applications, pages 53-61, 2001

19. B. Y. Su, J. Y. Ma, Y. S. Peng and M. Sheng, "Algorithm for GGBD Point De-noising and Simplification Based on K-means Clustering," Journal of System Simulation, pages 3-5, 2016

20. P. Tan, T. Fang, J. Xiao, P. Zhao and L. Quan, "Single Image Tree Modeling," ACM Transactions on Graphics, pages 1-7, 2008

21. F. Yan, A. Sharf, W. Lin, H. Huang and B. Chen, "Proactive 3D Scanning of Inaccessible Parts," ACM Transactions on Graphics, pages 1-8, 2014

22. L. L. Zeng, W. Zhang, Y. Yang, et al. "Multiple Density Leaf Reconstruction Based on Limited Details." Computer Science,pages 3-4, 2016 
Lanling Zeng is currently working as associate professor and director in the department of Computer Science and Communication Engineering at Jiangsu University, Zhenjiang, China. She received her B.E. in the department of telecommunications from Lanzhou University in 1996 and M.E. in the department of computer science from Shenyang Ligong University in 2006. Also she has been awarded her Ph.D. in applied mathematics from Zhejiang University in 2009. Her research interests mainly in Computer graphics. She has twenty years of teaching and research experience.

Lingling Zhang is a master student from the department of Computer Science and Communication Engineering, Jiangsu University in Zhenjiang, China. Her research interests mainly include Computer graphics.

Yang Yang is in the department of Computer Science, University of Science and Technology of China, Hefei, Anhui Province, China, Doctor of Computer Science and Technology, 2012. And he is in the department of Computer Science, Anhui University of Finance and Economics, Bengbu, Anhui Province, China, Bachelor of Computer Science and Technology, 2007. He is a professor in School of Computer Science and Telecommunication Engineering, Jiangsu University now. His research interests include Computer graphics and Computer animation.

Wei Zhang is currently working as a teacher in the department of Information and Communication at Jiangsu Wuzhong Secondary Vocational School. She received her B.E. in the department of Computer Science and Communication Engineering at Jiangsu University and M.E. in the department of Computer Science and Communication Engineering at Jiangsu University. Her research interests mainly include Computer graphics.

Yongzhao Zhan received his bachelor degree in Computer Software from Fuzhou University, his M.S. degree in Computer Science from Jiangsu University, and his Ph.D. degree in Computer Science from Nanjing University, China, in 1984, 1990 and 2000 respectively. From October 1995 to October 1996, He was at Konstanz University of Technology, Germany, as a Visiting Scholar. From June to August 2009, He was at University of Konstanz, Germany, as a Senior Visiting Scholar. He is currently a Professor of School of Computer Science and Communication Engineering at Jiangsu University. His research interests include human computer interaction, distributed computer system, and multimedia technology. He has led more than 10 national or provincial and ministerial level research projects, published more 200 papers and 6 books. He got 6 science and technology advantage awards of provincial and ministerial level. 